International Journal of Distributed and Parallel Systems (IJDPS) Vol.2, No.6, November 2011

\title{
REDUCING PAPR OF OFdM BASEd WiRELESS SYSTEMS USING COMPANDING WITH CONVOLUTIONAL CODES
}

\author{
Pawan Sharma ${ }^{1}$ and Seema Verma ${ }^{2}$ \\ ${ }^{1}$ Department of Electronics and Communication Engineering, Bhagwan Parshuram \\ Institute of Technology, Rohini, New Delhi, India \\ Pawan061971@yahoo.co.in \\ ${ }^{2}$ Department of Electronics and Communication Engineering, Banasthali University, \\ Rajasthan, India \\ seemaverma3@yahoo.com
}

\begin{abstract}
OFDM is a promising broadband technique. However, the implementation disadvantage of OFDM is Peak-to-Average Power Ratio (PAPR) which outweighs all the potential benefits of OFDM. In this paper, a new scheme will be introduced, that combines selective mapping and companding technique to reduce PAPR. However, companding technique is a distortion technique but by using convolutional codes with companding also improves BER performance of the OFDM system. It also removes the disadvantage of SLM technique of extra side information i.e. this technique does not require any side information unlike in conventional SLM. By considering the example of OFDM with BPSK modulation, it is shown in simulation results that the proposed scheme performs well in both reducing PAPR and improving BER performance without the need of any side information. It has been calculated that PAPR of OFDM signal can be reduced by $5 \mathrm{~dB}$ over the original system with the proposed scheme.
\end{abstract}

\section{KEYWORDS}

Orthogonal Frequency Division Multiplexing (OFDM), Peak-To-Average Power Ratio (PAPR), Selective Mapping (SLM), Bit Error Rate (BER), Complementary Cummulative Distribution Function (CCDF)

\section{INTRODUCTION}

Orthogonal frequency division multiplexing (OFDM) has been recently gaining much popularity in wireless and wire line applications. For wireless communications, an OFDMbased system can provide greater immunity to multi-path fading and reduce the complexity of equalizers [1]. Now OFDM have been included in digital audio/video broadcasting (DAB/DVB) standard in Europe, and IEEE 802.11, IEEE 802.16 wireless broadband access systems, etc. OFDM is a multicarrier modulation technique which splits high-rate data streams into a number of lower data rate streams that are transmitted simultaneously over a number of subcarriers. Because the symbol duration increases for the lower rate parallel subcarriers, the relative amount of dispersion in time caused by multipath delay spread is decreased. Inter symbol interference (ISI) is eliminated almost completely by introducing a guard time in every OFDM symbol [2]. Moreover, OFDM provides greater immunity to multi-path fading and impulse noise, and reduces the complexity of equalizers, while efficient hardware implementation can be realized using Fast Fourier Transform (FFT) techniques. In despite of its many advantages over other techniques, there is a serious problem of peak-to-average power ratio (PAPR) of the OFDM signal [3] which causes its practical implementation difficult. This problem arises from the nature of the modulation itself, where multiple sub-carriers are added together to form the 
signal to be transmitted. Usually, the systems are constrained to a limited peak power due to the limitation of the dynamic range over which the transmitter amplifier operates linearly. Several schemes have been proposed for reducing PAPR, such as clipping and filtering technique [4], coding [5], partial transmit sequences(PTS) technique [6], selective mapping(SLM) technique [7] and coding techniques [8]. On the basis of their performance, they are classified under two categories i.e. distortion technique and distortion less technique. In this paper, we proposed a scheme that uses companding technique to reduce the PAPR. But companding is a distortion technique that increases its BER at a particular SNR. This problem is rectified by combining convolutional code with companding technique. This scheme will be compared with the original system and all the systems will be evaluated under the effect of additive white Gaussian noise (AWGN).

The organization of this paper is as follows. Section 2 presents the PAPR problem in OFDM systems. Proposed scheme is introduced in section 2.1. Algorithms are shown in section 2.2. Simulation results are reported in section 2.3 and conclusions are presented in section 3.

\section{OFDM SYSTEM MODEL AND PAPR PROBLEM}

In conventional OFDM systems, whole system bandwidth is divided into many orthogonal subcarriers with narrow bandwidth, and data blocks are transmitted independently on the subcarriers. In the discrete time domain, an OFDM signal $x_{n}$ of $N$ subcarriers can be expressed as

$$
x_{n}=\frac{1}{\sqrt{N}} \sum_{k=0}^{N-1} X_{k} e^{j 2 \pi k n / N} \quad, \quad 0 \leq n \leq N-1
$$

Where $X_{k}, \mathrm{k}=0,1,2,3 \ldots . ., \mathrm{N}-1$, are input symbols modulated by BPSK, QPSK or QAM and $n$ is the discrete time index.

The PAPR of an OFDM signal is defined as the ratio of the maximum to the average power of the signal, as follows

$$
\operatorname{PAPR}(x)=10 \log _{10} \frac{\max \left\{\left|x_{n}\right|^{2}\right\}}{E\left\{|x|^{2}\right\}} \quad 0 \leq n \leq N-1
$$

Where $\mathrm{E}\{$.$\} denotes the expected value operation and x=\left[x_{1}, x_{2}, x_{3}, \ldots \ldots x_{N-1}\right]^{T}$

In the worst case all the sub-carriers may add together to form the signal due to which amplifier is no longer operates in its linear dynamic range and this causes serious problems due to nonlinearity of the power amplifiers.

When the OFDM signal with high PAPR passes through a non-linear device, (power amplifier working in the saturation region), the signal will suffer significant non-linear distortion [9]. This non-linear distortion will result in in-band distortion and out-of-band radiation. The in-band distortion causes system performance degradation and the out-of-band radiation causes adjacent channel interference (ACI) that affects systems working in the neighbour bands. To lessen the signal distortion, it requires a linear power amplifier with large dynamic range. However, this linear power amplifier has poor efficiency and is so expensive. Hence, it is necessary to reduce the peak to average power ratio of the OFDM signal as it outweighs all the potential benefits of an OFDM system. Usually, Complementary Cumulative Distribution Function (CCDF) can be used to evaluate the performance of any PAPR reduction schemes, given by [10]

$$
\operatorname{CCDF}\left(N, P A P R_{0}\right)=1-\left(1-e^{-P A P R_{0}}\right)^{N}
$$




\subsection{PROPOSED SCHEME}

In this paper we present a scheme that uses companding technique to reduce PAPR of OFDM signal. Companding means compressor and expander. At the transmitter, the dynamic range of the signal is compressed using compressor. Here, we are using u-law compressor which is defined by

$$
V=\frac{M \log _{e}\left(1+\frac{\mu|m|}{M}\right)}{\log _{e}(1+\mu)} \operatorname{sgn}(m)
$$

Where $\mathrm{m}$ and $\mathrm{v}$ are the normalized input and output voltages, and $\mathrm{u}$ is a positive constant. The dynamic range capability of the compressor improves with increasing $u$ but the SNR for low level signals increases at the expense of snr for high level signal. To accommodate these two conflicting requirements, a compromise is made in choosing the value of parameter $u$. for given value of $u$ the derivative of $m$ with respect to $v$ is defined by

$$
\frac{d|m|}{d|v|}=\frac{\log (1+\mu)}{\mu}(1+\mu|m|)
$$

To restore the signal to its relative level, we use a device at receiver which owns characteristics complementary to the compressor and is known as expander. Its equation is given by

$$
m=\frac{M}{\mu}\left(e^{|v| \log (1+\mu) / M}-1\right) \operatorname{sgn}(V)
$$

Although, companding is quite effective in reducing peak-to-average power ratio of the OFDM signal. But it is a distortion technique and degrades bit error rate performance of the system. Hence, we employ convolutional codes with companding to improve the system performance.

\subsection{ALGORITHM FOR TRNASMITTER}

The input data block X1, X2 ....Xn are convolutionally encoded with code rate $=1 / 2$ and constraint length $=7$.

1. The coded data bits are mapped using BPSK modulation technique, and then take IFFT of the modulated data to form OFDM symbols.

$\mathrm{X}_{1}$

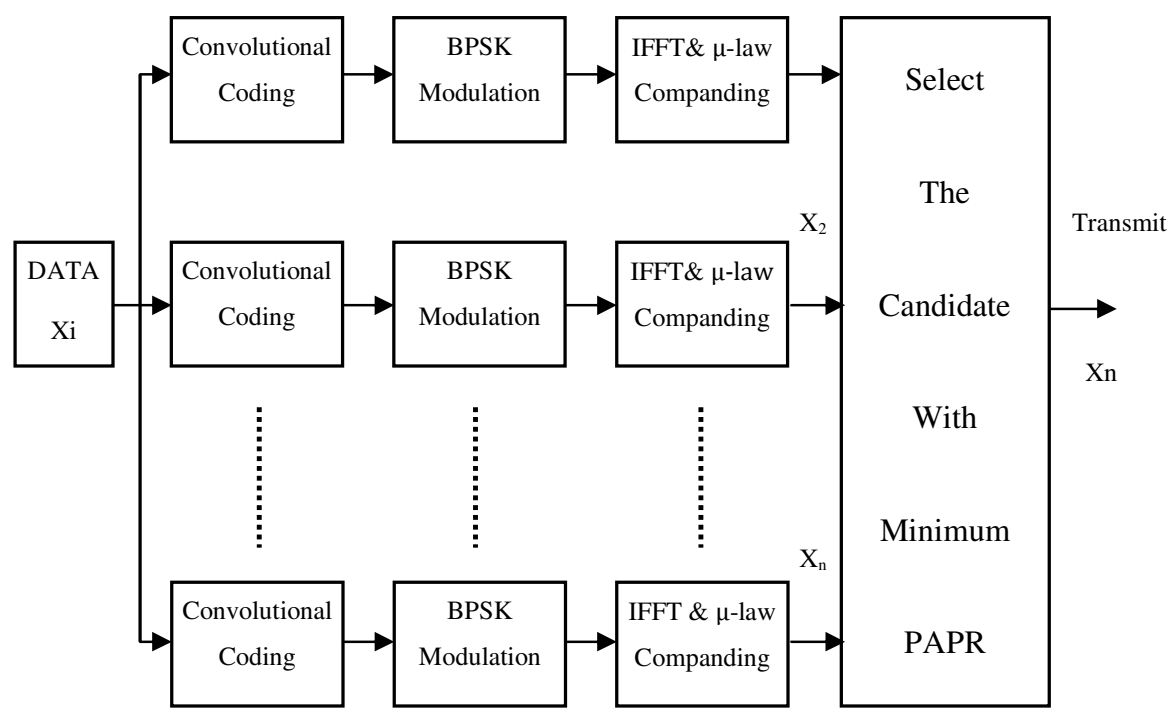

Figure1.Transmitter

2. These symbols are compressed using $\mu$-law compressor before transmitting the data. 
International Journal of Distributed and Parallel Systems (IJDPS) Vol.2, No.6, November 2011

3. Repeat step 1 to step 3 for number of times to produce different number of candidates for each data block.

4. Select the candidate with minimum PAPR for transmission. Plot the CCDF curve for different values of $\mu$.

\section{ALGORITHM FOR RECEIVER}

1. Introduce additive white Gaussian noise in the transmitted signal.

2. Select the candidate with different values of PAPR and plot the CCDF curve for the different values of $\mu$.

3. After expanding the signal, FFT of the signal is computed.

4. Demodulate the data points using BPSK demodulation.

5. The data bits are convolutionally decoded using Viterbi algorithm with hard decoding.

6. Compare the output data points with input data points and calculate the bit error rate.

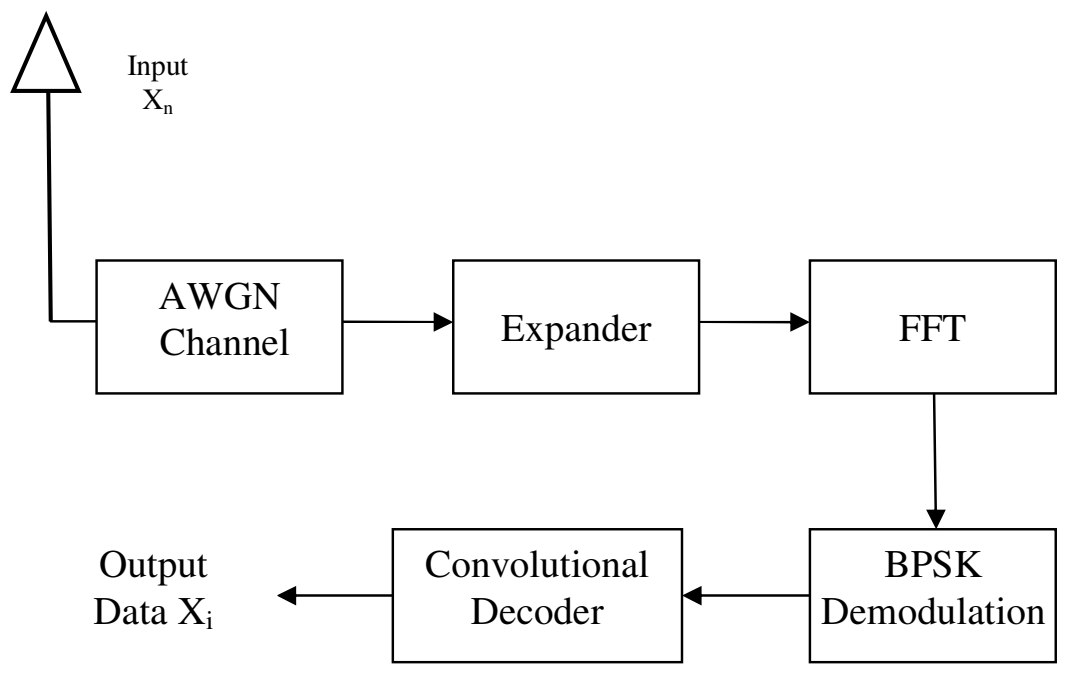

Figure2.Receiver

\subsection{SIMULATION RESULTS}

In this section, computer simulations are used to evaluate the peak-to-average ratio reduction capability and bit error rate with proposed scheme. In simulation, an OFDM system is considered with subcarriers $N=128$ and BPSK modulation. We can evaluate the performance of PAPR using the complementary cummulative distribution function for PAPR of OFDM signal. The cumulative distribution function (CDF) is also one of the most regularly used parameters, which is used to measure the efficiency of PAPR technique. The CDF of the amplitude of a signal sample is given by

$F(z)=1-\exp (z)$

However, the complementary CDF (CCDF) is used instead of CDF, which helps us to measure the probability that the PAPR of a certain data block exceeds the given threshold. The CCDF of the PAPR of the data block is desired is our case to compare outputs of various reduction techniques. This is given by 
$P(P A P R>Z)=1-P(P A P R>Z)=1-(1-\exp (-2))^{N}$

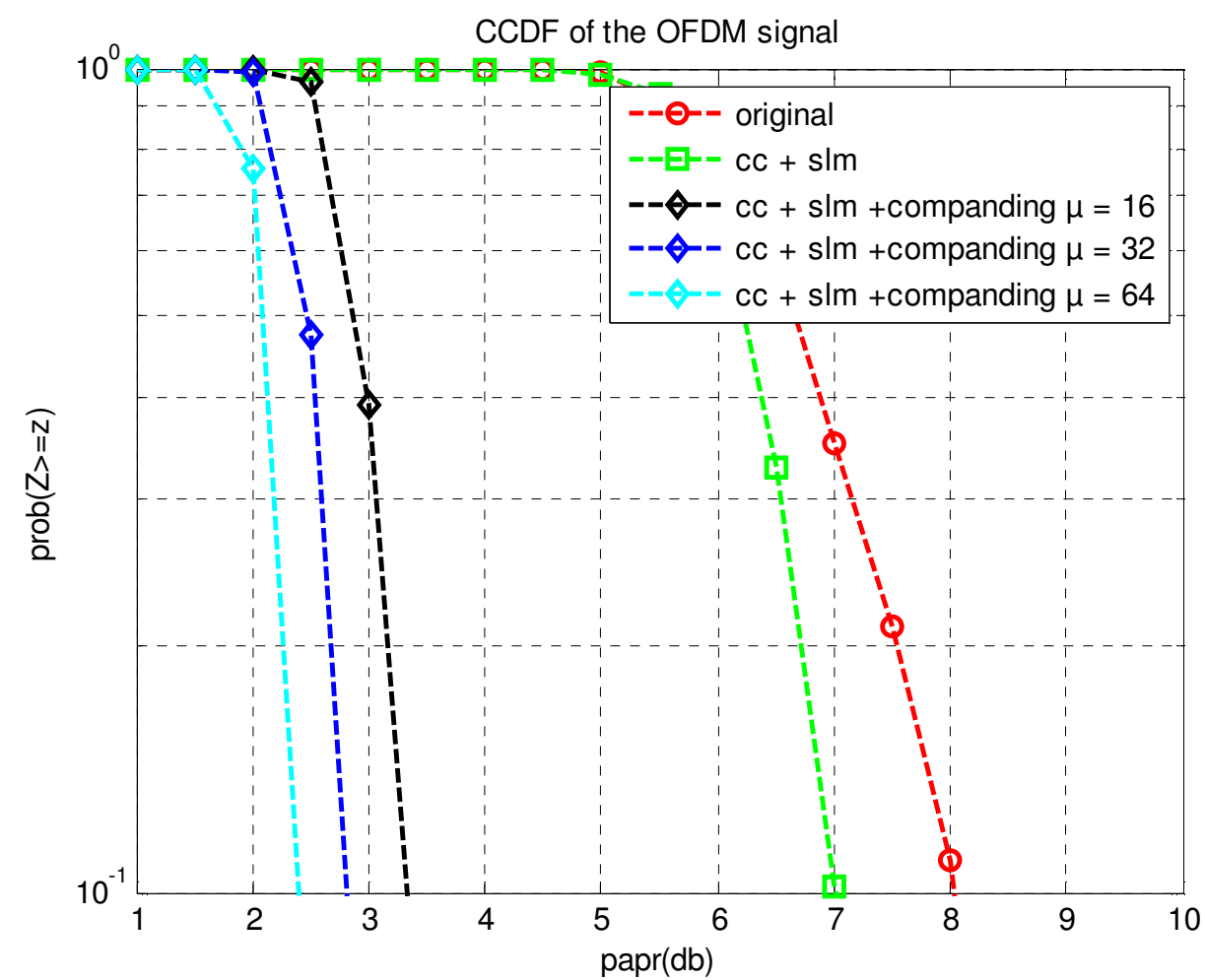

Figure3. CCDF for the convolutionally coded OFDM signal for different values of ' $\mu$ '

Figure. 3 shows the CCDF performance of the proposed scheme about the PAPR reduction. In simulation, we have taken different values of ' $\mu$ '. We can see that when convolutional codes and companding are combined, this technique provides better performance than that of the simple convolutional coding with SLM. At CCDF $=10^{-1}$, the PAPR of proposed scheme is almost $3 \mathrm{~dB}$ smaller than that of convolutional coding technique. The companding with convolutional coding may reduce approximately 5.5 dB PAPR compared to that of original OFDM signal. 


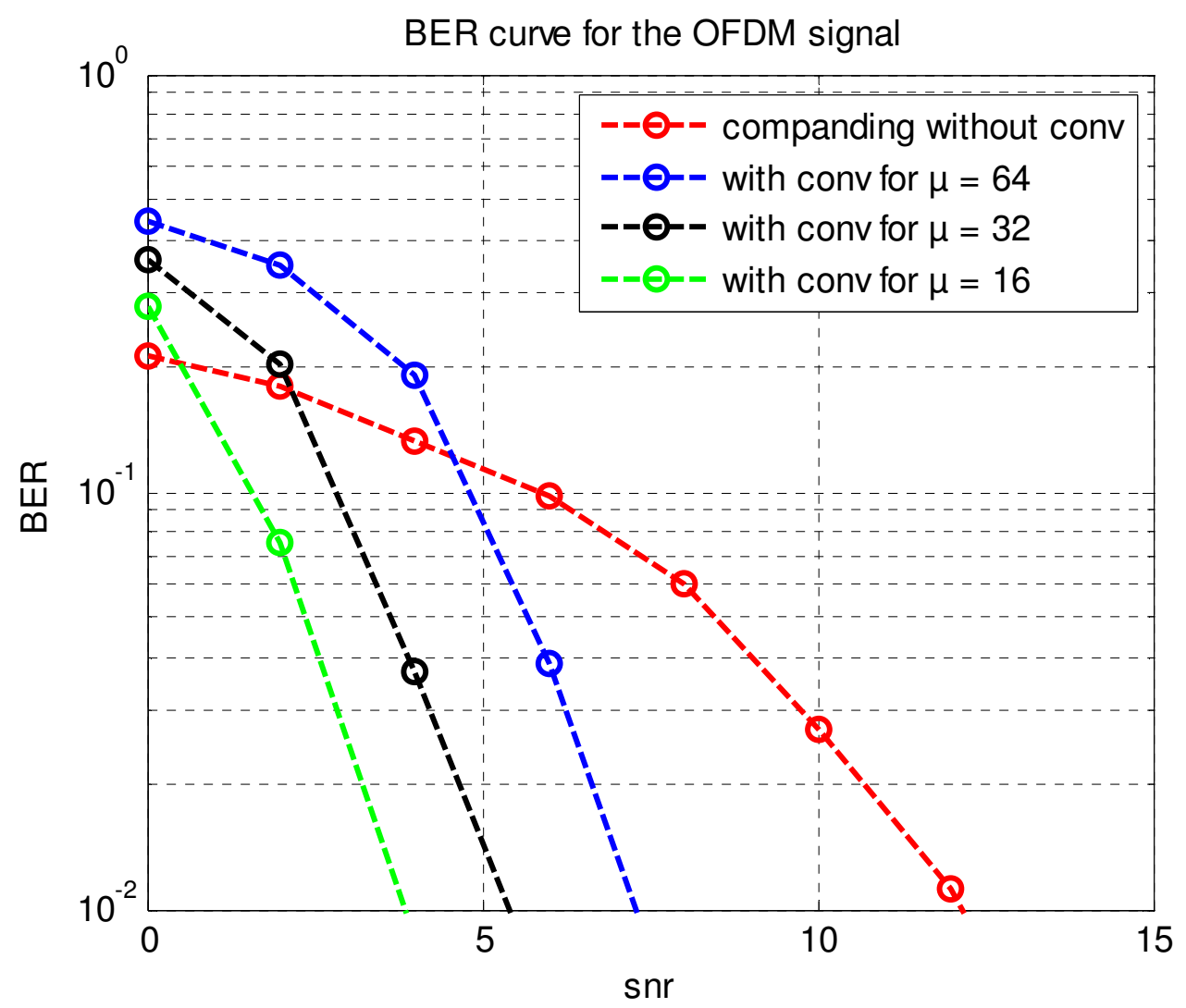

Figure4. BER for the convolutionally coded OFDM signal for different values of ' $\mu$ '

\section{Conclusions}

In this paper, a PAPR reduction scheme for the OFDM signal based on companding and convolutional coding technique has been proposed. The PAPR reduction and BER performance have been evaluated by computer simulation. Simulation results shows that the PAPR reduction performance is improved by using proposed scheme as compared with using companding technique only. On the other hand, the BER of system using proposed PAPR reduction scheme is not degraded.

\section{REFERENCES}

1. S.H.Han and J.H.Lee, "An Overview of Peak-to-Average Power Ratio Reduction Techniques For Multicarrier Transmission," IEEE Wireless Communications, Vol.12, No.2, Apr.2005, pp.56-65.

2. C.L.Wang, M.Y.Hsu, and Y.Wuynag, "A Low-Complexity Peak-to-Average Power Ratio Reduction Technique for OFDM systems," Global Telecommunication Conference, 2003 Globecom'03 IEEE, pp.2375-2379.

3. T.Jiang and Y.Wu, "An Overview: Peak-to-Average Power Ratio Reduction Techniques for OFDM Signals,” IEEE Transactions on Broadcasting, Vol.54, No.2, June 2008, pp.257-268.

4. K.D.Rao and T.S.N.Murthy, "Analysis of Effects of Clipping and Filtering on the Performance of MB-OFDM UWB Signals," Proc. of the $200715^{\text {th }}$ International Conference on Digital Signal Processing (DSP 2007), IEEE, pp.559-562.

5. R.F.H.fischer and C.Siegl, "Reed-Solomon and Simplex Codes for Peak-to-Average Power Ratio reduction in OFDM,'IEEE trans.on Information Theory, Vol.55,No.4, Apr.2009, pp.1519-1528. 
International Journal of Distributed and Parallel Systems (IJDPS) Vol.2, No.6, November 2011

6. A.Ghassemi and T.A. Gulliver, "PAPR Application of OFDM Using PTS and Error-Correcting Code Subblocking," IEEE Trans. On Wireless Communications, Vol.9, No.3, March 2010, pp.980-989.

7. A.D.S.Jayalath and C.Tellambura, "SLM and PTS Peak-Power Reduction of OFDM Signals without side information," IEEE Transactions on Wireless Communications, Vol.4, Sept.2005, pp.2006-2013.

8. Abdulla A. Abouda, "PAPR reduction of OFDM signal using Turbo coding and selective mapping", pp.239-241, Proc. Of NORSIG, (2004).

9. A.D.S.Jayalath and C.Tellambura, "SLM and PTS Peak-Power Reduction of OFDM Signals without side information," IEEE Transactions on Wireless Communications, Vol.4, Sept.2005, pp.2006-2013.

10. H. Ochiai and H. Imai, "On the distribution of the peak-to-average power ratio in OFDM signals,” pp. 282-289, IEEE Trans. Commun., vol. 49, (2001). 